rq

تأثير هرمون IBA) Apiren ومواد نتروجينية) على النمو الخضري والتبكيربالإز هار وعقد الفية

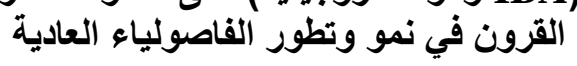

إبراهيم الثتيوي

قسم البساتين - كلية الزراعة - جأمعة الفرات- سوريا

لقد تم إجر اء البحث على أربعة أصناف من الفاصوليا العاديـة: strike, I golamska,

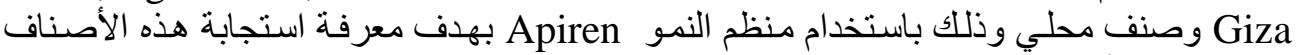

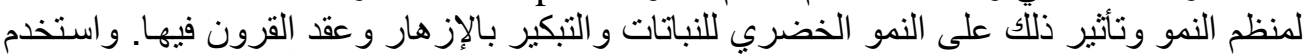

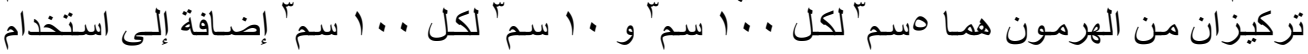

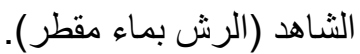

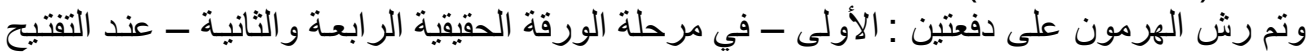

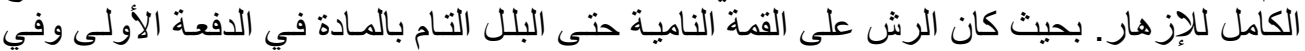

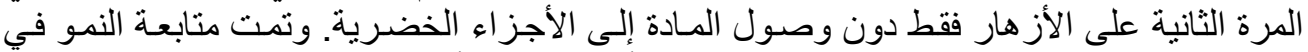

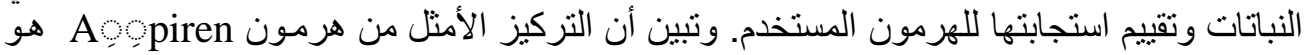

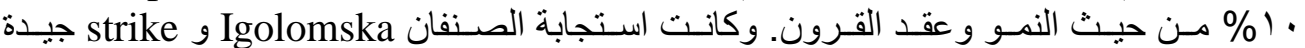
لاستخدام الهرمون و أفضل من بقية الأصناف مما يوصى بـ بزر اعتها في المنطقة الشرقية من سوريا.

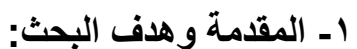

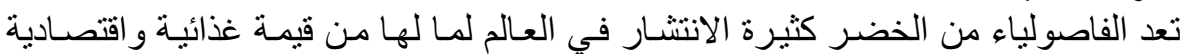

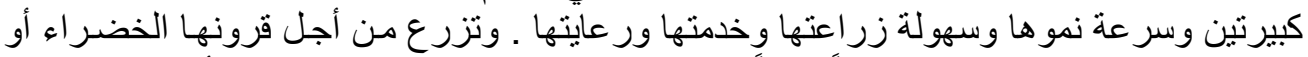

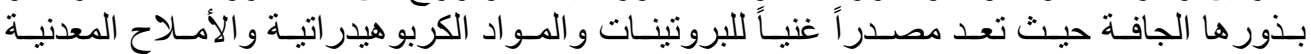

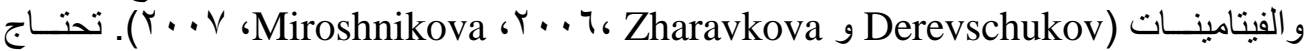

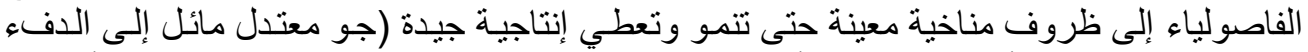

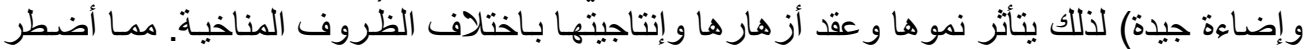

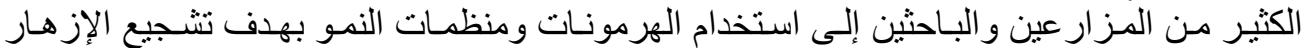

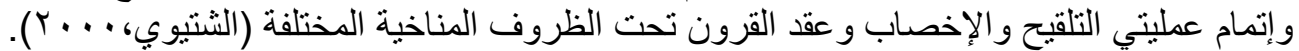

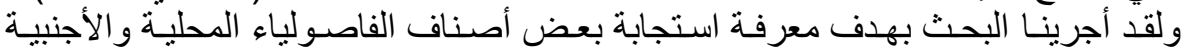

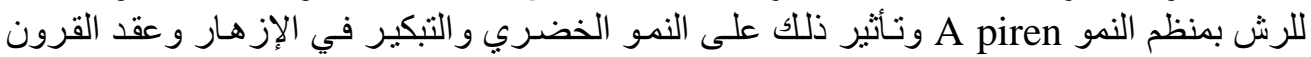

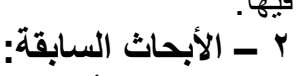

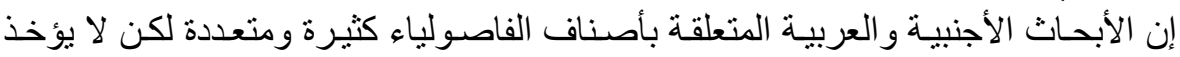

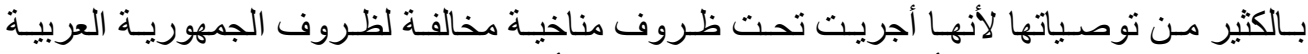

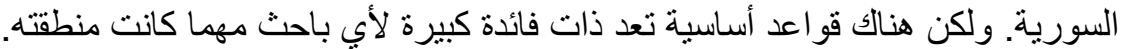

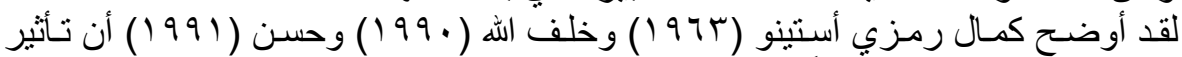

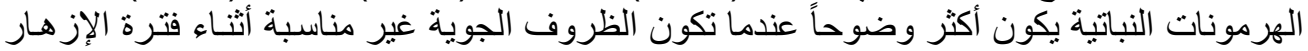

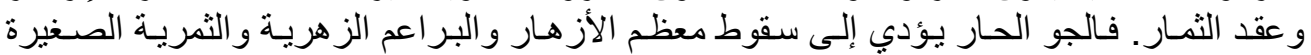

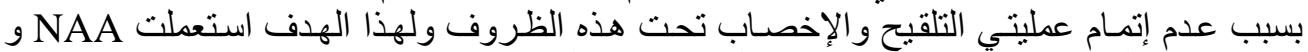

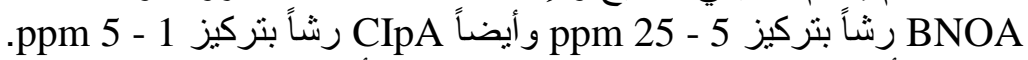

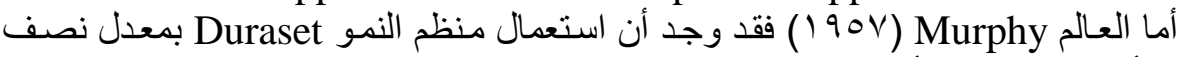
رطل للفدان أو 100 ppm أوكسين 2,4,5-T في الفاصولياء ساعد في زيادة القرون وتحسين نسبة

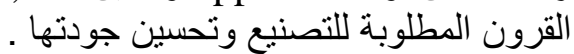

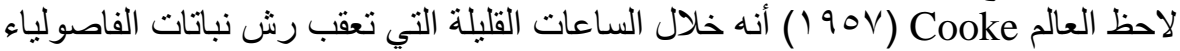

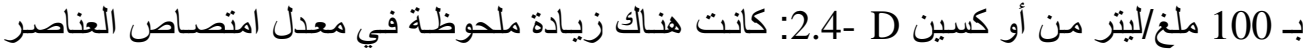

Fayoum J. Agric. Res. \& Dev., Vol.23, No.2, July, 2009 
r.

المشعة من كلوريد البوتاسيوم وكبريتات الكالسيوم. وفي تجربة أخرى بالرش على الأوراق بـ -2.4 -

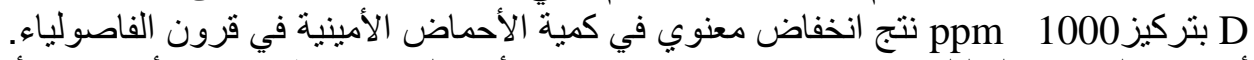

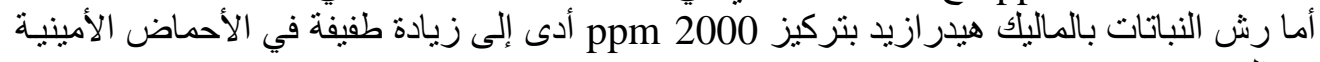
في القرون.

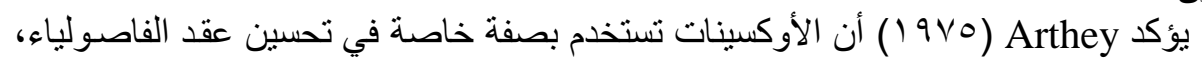
ويبين أن المعاملة بمركبات Alkylene diamines مثل ذات السلاسل الطويلة تفيد

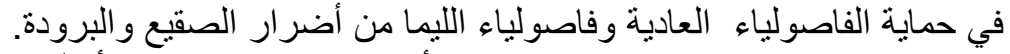

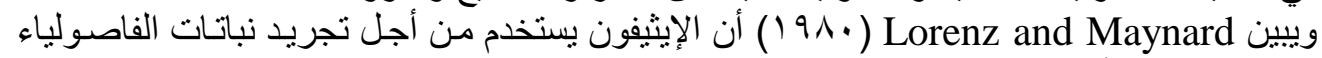

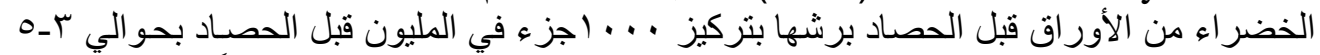

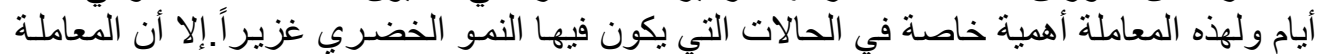

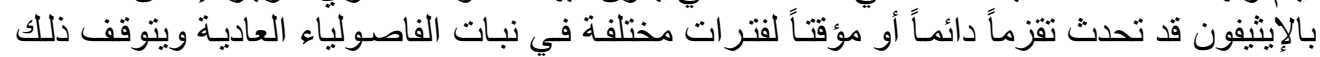

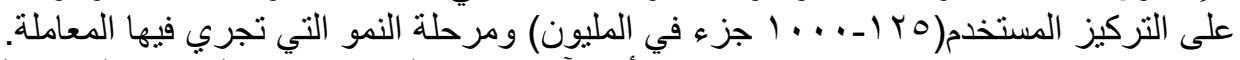

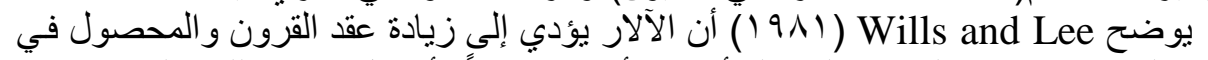

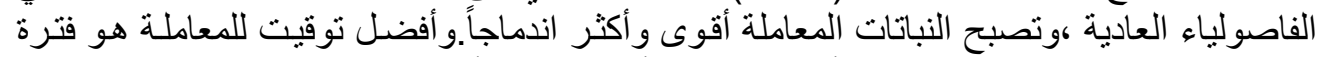

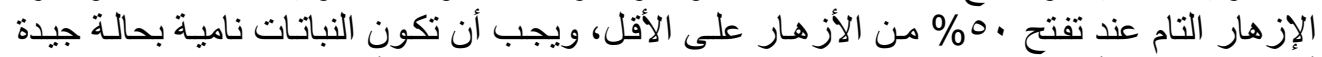

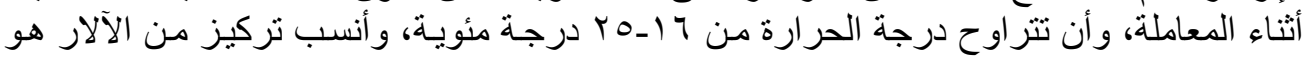
$\% \cdot 10$

يوجد بعض الهرمونـات ذات التـأثير السـلبي على النباتـات، و هذا مـا أوضـحه العـالم (1964) عندما اسـتخدم (Jeffreys

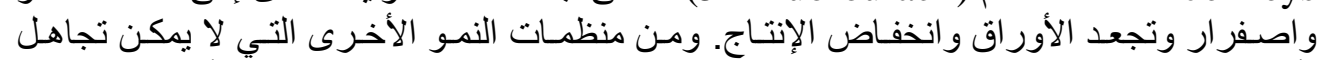

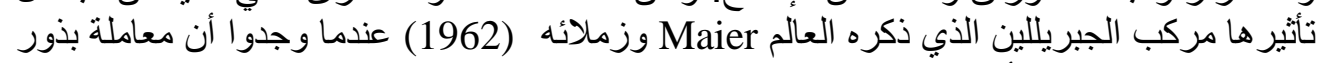

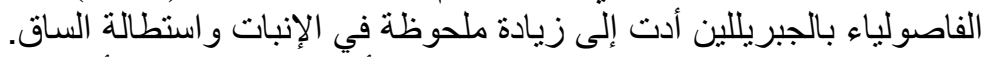

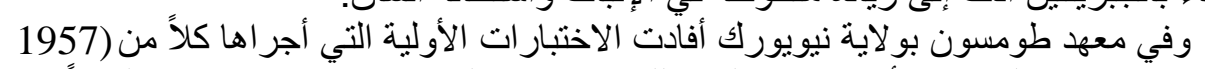

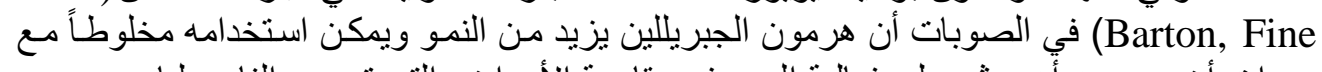

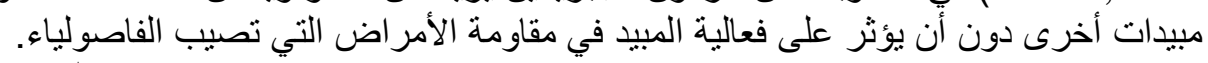

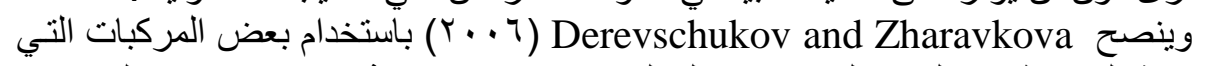

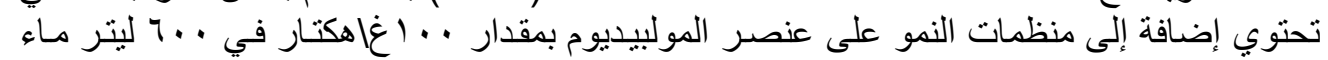
عند زراعة الفاصولياء العادية.

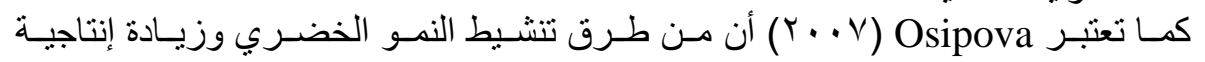

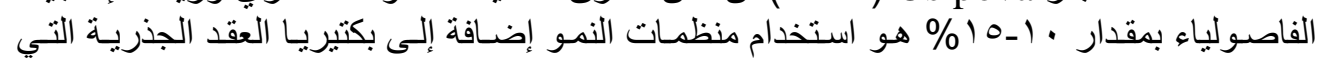

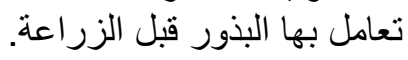

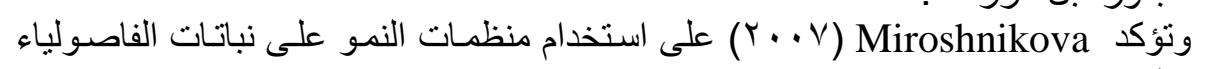

لزيادة نمو النباتات وتحسين إنتاجيتها.

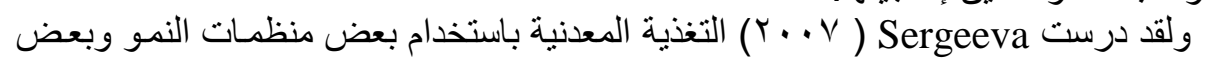

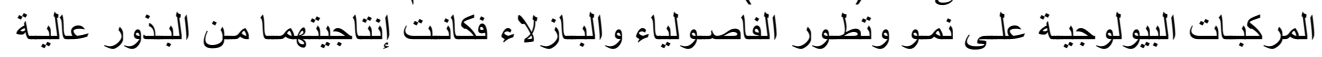

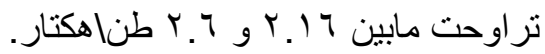
r- مواد وطر ائق البحث:

أجريت هذه الدر اسة في مزر عة خاصة لإثة للباحث ، بمدينـة دير الزور باستعمال أربع أصناف مـن

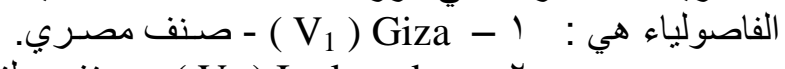
- ( $\left.\mathrm{V}_{2}\right)$ Igolomska ع ( ع -

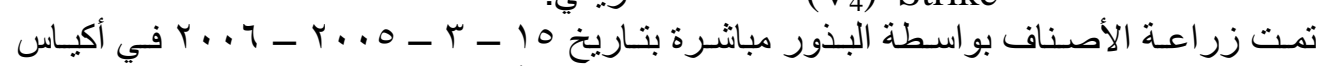

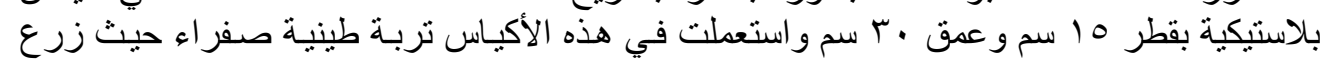

Fayoum J. Agric. Res. \& Dev., Vol.23, No.2, July, 2009 
ए

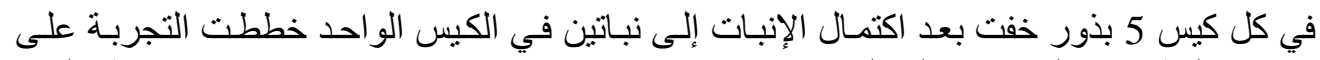

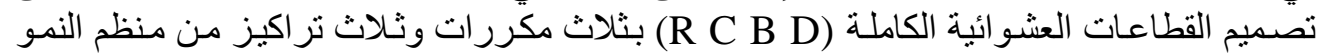
التجاري Apiren بحيث كانت المعاملات على الثمات الثكل التالي:

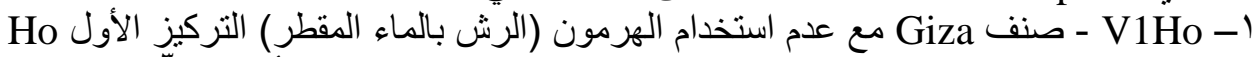

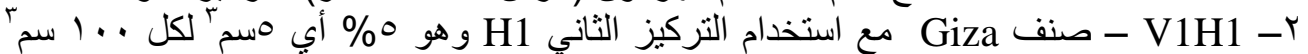
r- ك- Giza مع استخدام التركيز الثالث H2H2 ع - V2Ho

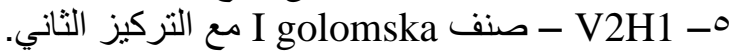

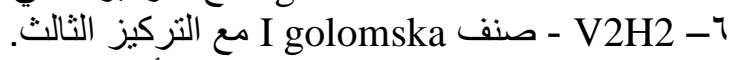

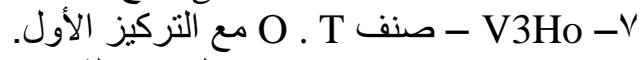

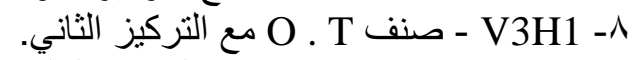

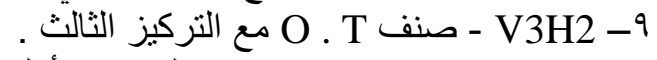
.

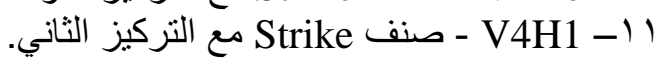

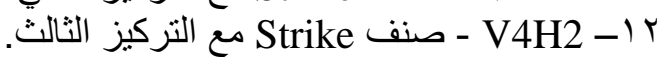

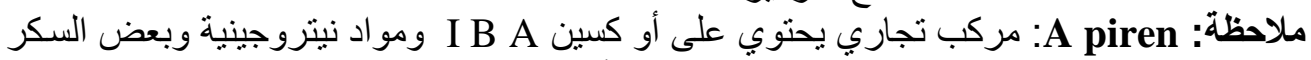

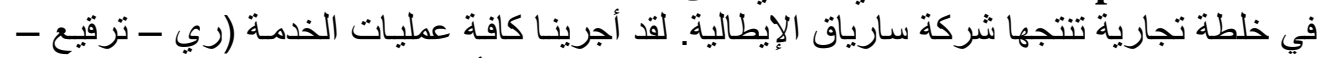

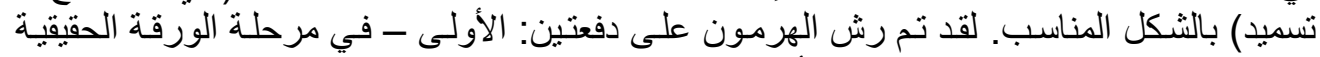

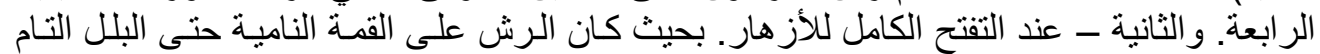

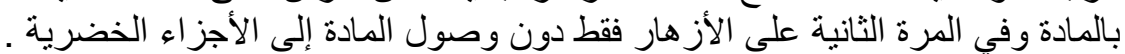

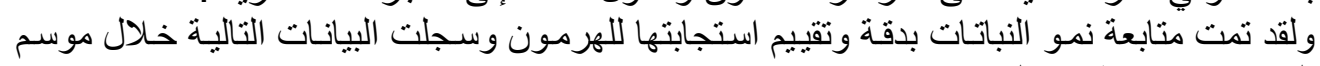

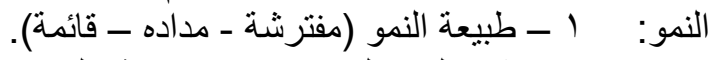

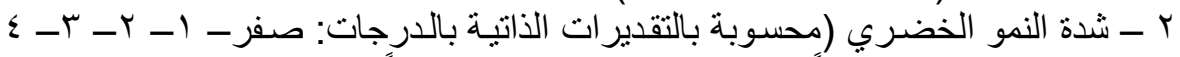

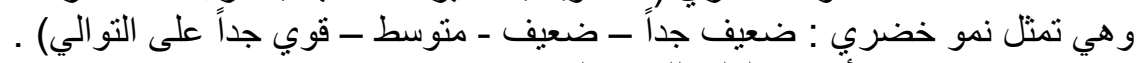

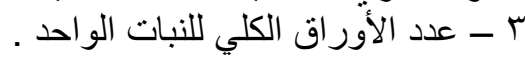

عـ مساحة الورقة : محسوبة بنظام الدوائر و الوزن النسبي و استعمال المعادلة التالية: مساحة الورقة

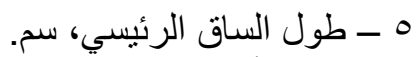

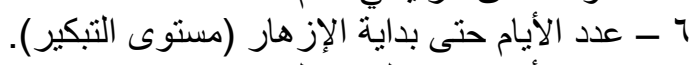

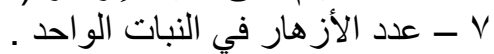

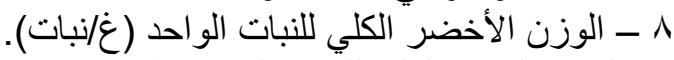

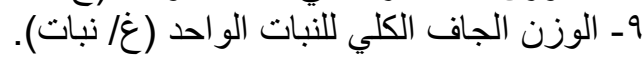

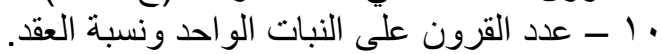

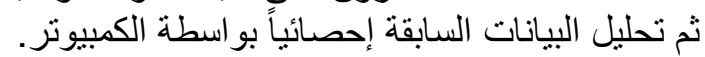

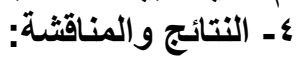

جدول (1): تأثنير تركيزات هرمون Apiren على النمو الخضري في أصناف الفاصولباء المختلفة في بداية الإزهار.

Fayoum J. Agric. Res. \& Dev., Vol.23, No.2, July, 2009 


\begin{tabular}{|c|c|c|c|c|c|c|}
\hline $\begin{array}{l}\text { طول الساق } \\
\text { (سم) }\end{array}$ & الأور اقد & مساحة & شذة النمو & طبيعة & تركيز & الأصناف \\
\hline 58.8 & 10.5 & 36.2 & 2.7 & مداد & Ho & \\
\hline 32.5 & 5.6 & 35.6 & 0.7 & مداد & $\% 5 \mathrm{H} 1$ & V1 \\
\hline 28.3 & 7.5 & 41.4 & 2.7 & مداد & $\% 10 \mathrm{H} 2$ & \\
\hline 39.9 & 7.8 & 37.7 & 2.0 & & & المتوسط \\
\hline 46.0 & 8.6 & 45.1 & 2.7 & قائم & Ho صفر & V2 \\
\hline 44.4 & 7.3 & 39.3 & 2.7 & قائر & \% $5 \mathrm{H} 1$ & \\
\hline 38.9 & 7.3 & 51.6 & 2.3 & قائم & $\% 10 \mathrm{H} 2$ & \\
\hline 43.1 & 7.7 & 45.3 & 2.6 & & & المتوسط \\
\hline 48.4 & 13.6 & 40.4 & 2.0 & قصير & Ho صفر & \\
\hline 63.5 & 14.0 & 34.0 & 1.3 & قصير & $\% 5 \mathrm{H} 1$ & V3 \\
\hline 39.6 & 14.6 & 40.0 & 1.7 & قصبر & $\% 10 \mathrm{H} 2$ & \\
\hline 50.5 & 14.1 & 38.2 & 1.7 & & & المتوسط \\
\hline 26.5 & 15.3 & 50.1 & 2.3 & قصير & Ho صفر & \\
\hline 30.8 & 6.6 & 46.3 & 1.0 & قصير & $\% 5 \mathrm{H} 1$ & V4 \\
\hline 23.1 & 6.6 & 48.3 & 2.7 & قصير & $\% 10 \mathrm{H} 2$ & \\
\hline 26.8 & 9.3 & 48.2 & 2.0 & & & المتوسط \\
\hline 25.1 & 3.5 & 11.3 & - & - & & LSD \\
\hline
\end{tabular}

يوضح الجدول ( (1) عدم تأثثير تركيزات الأيبرين على طبيعة النمو في أصناف الفاصولياء

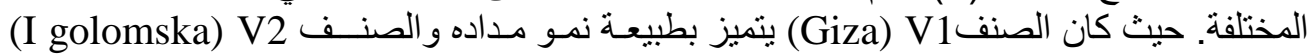

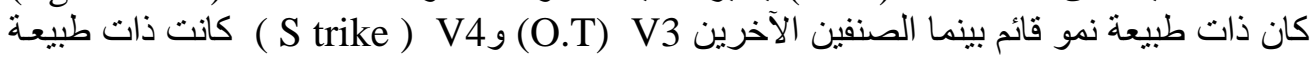

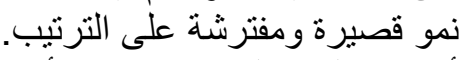

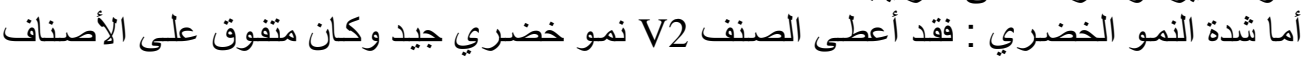

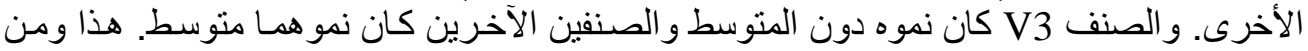

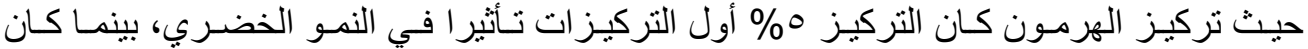

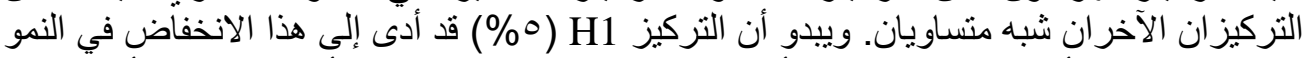

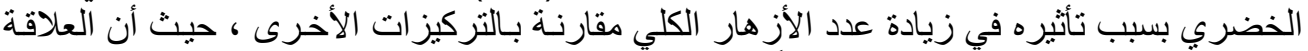

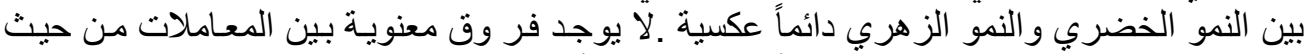

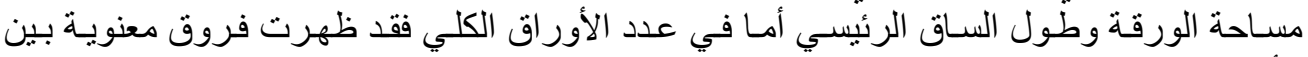

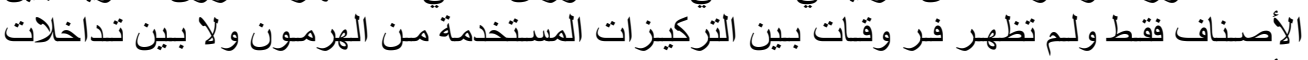

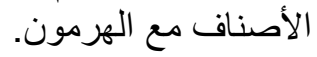

جدول (Y): الوزن الكلي لنباتات الفاصولياء متأثراً بالأصناف وتركيز ات هرمون A piren. 


\begin{tabular}{|c|c|c|c|}
\hline (جر الوزن الجات) & (جر الوز / نبات) & تركيزات الهرمون & الأصناف \\
\hline 2.1 & 10.3 & Ho & \multirow{3}{*}{$\begin{array}{c}\text { V1 } \\
\text { Giza }\end{array}$} \\
\hline 1.3 & 7.5 & $\% 5 \mathrm{H} 1$ & \\
\hline 1.7 & 7.3 & $\% 10 \mathrm{H} 2$ & \\
\hline 1.7 & 8.3 & & المتوسط \\
\hline 1.8 & 11.4 & Ho صفر Ho & \multirow{3}{*}{$\begin{array}{c}\text { V2 } \\
\text { I golomska }\end{array}$} \\
\hline 1.8 & 8.3 & $\% 5 \mathrm{H} 1$ & \\
\hline 1.2 & 9.2 & $\% 10 \mathrm{H} 2$ & \\
\hline 1.6 & 9.6 & & المتوسط \\
\hline 2.1 & 7.0 & Ho صفر & \multirow{3}{*}{$\begin{array}{l}\text { V3 } \\
\text { O.T }\end{array}$} \\
\hline 1.8 & 9.4 & $\% 5 \mathrm{H} 1$ & \\
\hline 1.5 & 8.5 & $\% 10 \mathrm{H} 2$ & \\
\hline 1.8 & 8.3 & & المتوسط \\
\hline 1.4 & 10.3 & Ho صفر Ho & \multirow{3}{*}{$\begin{array}{c}\mathrm{V} 4 \\
\text { S trick }\end{array}$} \\
\hline 1.3 & 6.7 & $\% 5 \mathrm{H} 1$ & \\
\hline 1.6 & 7.2 & $\% 10 \mathrm{H} 2$ & \\
\hline 1.4 & 8.1 & & المتوسط \\
\hline 0.6 & 1.9 & & $\% 5$ L S D \\
\hline
\end{tabular}

كما هو واضح من الجدول لا توجد فروق معنوية بين الأصناف أو التركيزات المختلفة للأبرين أو جدول (r): مستوى التبكير في الإزهار متأثراً بالأصناف وتركيزات الهرمون:

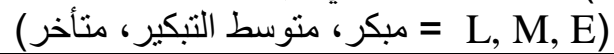

\begin{tabular}{|c|c|c|c|}
\hline \multicolumn{3}{|c|}{ تركيزات الهرمون } & \multirow{2}{*}{ الصنف } \\
\hline$\% 10 \quad \mathrm{H} 2$ & $\% 5 \mathrm{H1}$ & Ho & \\
\hline $\mathrm{M}$ & $\mathrm{L}$ & $E$ & Giza V1 \\
\hline $\mathrm{E}$ & $\mathrm{E}$ & $\mathrm{E}$ & I golomska V2 \\
\hline $\mathrm{L}$ & $\mathrm{E}$ & $\mathrm{M}$ & O. T V3 \\
\hline $\mathrm{E}$ & $E$ & $E$ & strike V4 \\
\hline
\end{tabular}

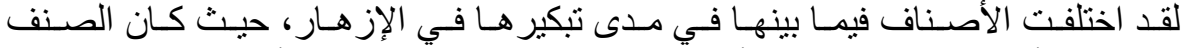

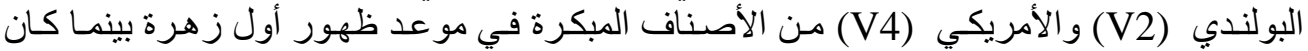

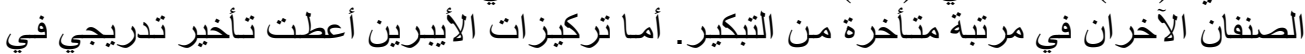

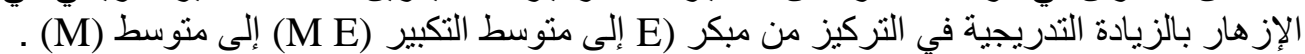

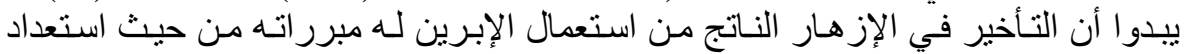

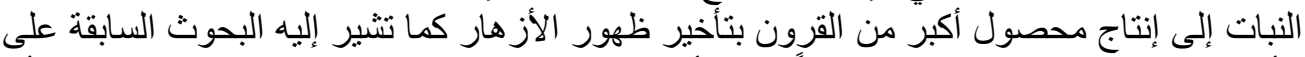

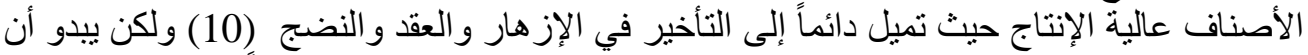

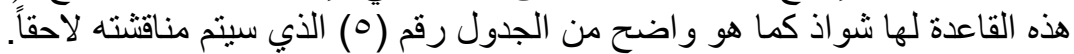

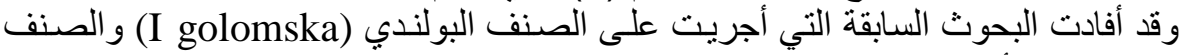

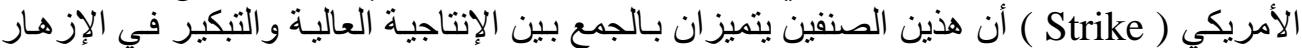
معاً كما ظهر من هذا البحث أبضاً. جدول (ء): عدد الأز هار الكلي في النبات الواحد لأصناف الفاصولياء المعاملة بهرمون Apiren .

Fayoum J. Agric. Res. \& Dev., Vol.23, No.2, July, 2009 


\begin{tabular}{|c|c|c|c|c|}
\hline \multirow{2}{*}{ المتوسط } & \multicolumn{3}{|c|}{ التركيز ات } & \multirow{2}{*}{ الصنف } \\
\hline & $\% 10 \mathrm{H} 2$ & $\% 5 \mathrm{H} 1$ & Ho & \\
\hline 6.3 & 5.3 & 6.3 & 8.0 & Giza V1 \\
\hline 18.5 & 25.3 & 22.6 & 7.6 & I golomska V2 \\
\hline 10.8 & 11.6 & 12.0 & 9.6 & O.T V3 \\
\hline 13.2 & 11.6 & 12.6 & 15.6 & strike V4 \\
\hline 4.8 & & & & $5 \%$ LSD \\
\hline
\end{tabular}

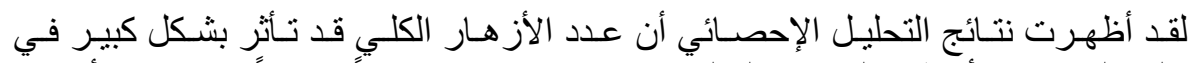

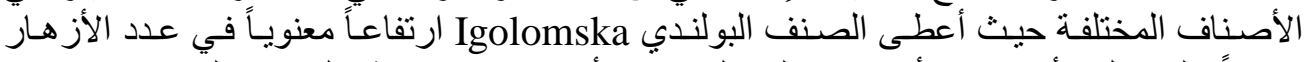

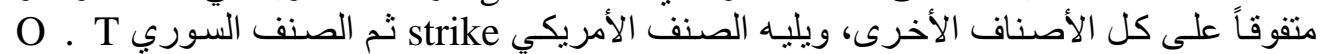

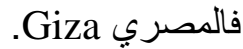

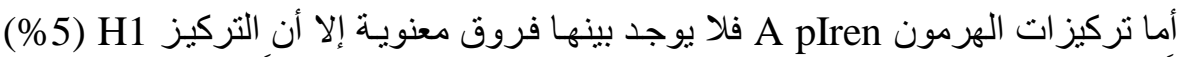

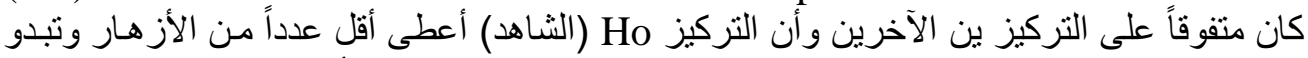

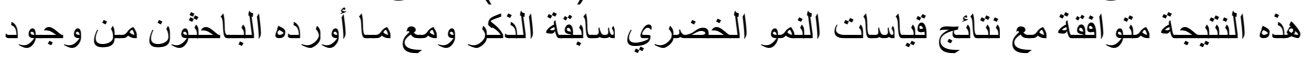

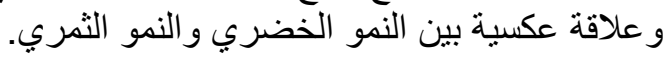

جدول (0): عدد القرون الكلي للنبات الواحد في أصناف الفاصولياء المختلفة بعد أسبو عين من بداية

\begin{tabular}{|c|c|c|c|c|}
\hline \multirow{3}{*}{ المتوسط } & \multirow{2}{*}{\multicolumn{3}{|c|}{ تركيز هرمون A preen }} & الإز هار \\
\hline & & & & \multirow{2}{*}{ الصنف } \\
\hline & $\% 10 \mathrm{H} 2$ & $\% 5 \mathrm{H} 1$ & صفر H0 & \\
\hline 1.7 & 2.7 & 1.3 & 1.0 & (V1) Giza \\
\hline 3.1 & 4.3 & 4.0 & 1.0 & (V2) Igolomska \\
\hline 2.1 & 3.3 & 2.0 & 1.0 & (V3) O.T \\
\hline 2.8 & 3.3 & 2.7 & 2.3 & (V4) strike \\
\hline 0.15 & - & - & - & $\% 5 \mathrm{LSD}$ \\
\hline
\end{tabular}

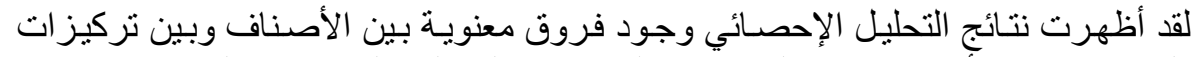

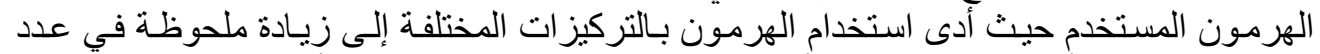

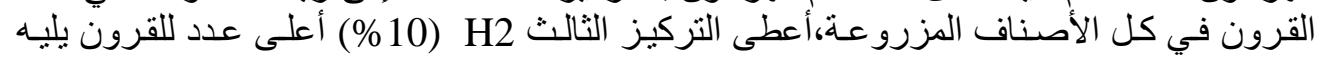

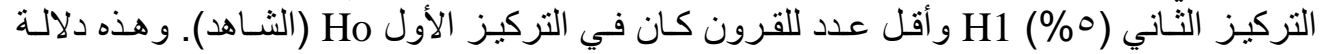

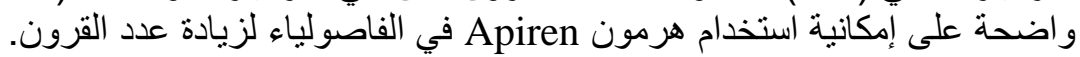

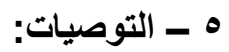

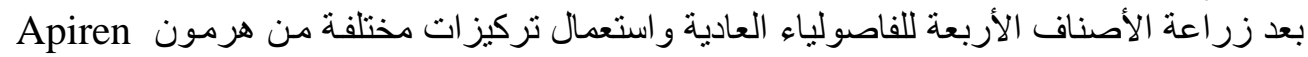

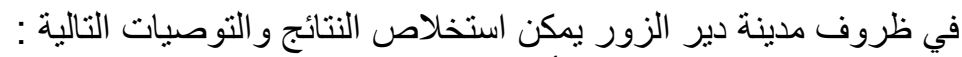

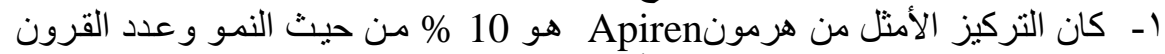

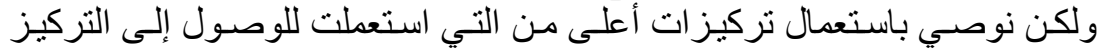

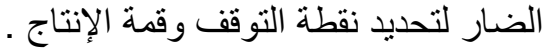

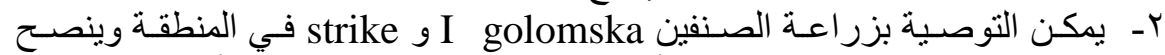

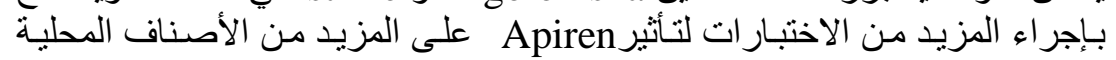
و المستوردة . r- إن هرمون Apiren منظم جيد ينصسح باستعماله في جميع زر زاعـات الفاصولياء

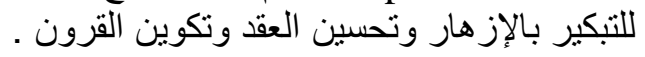

Fayoum J. Agric. Res. \& Dev., Vol.23, No.2, July, 2009 
ro

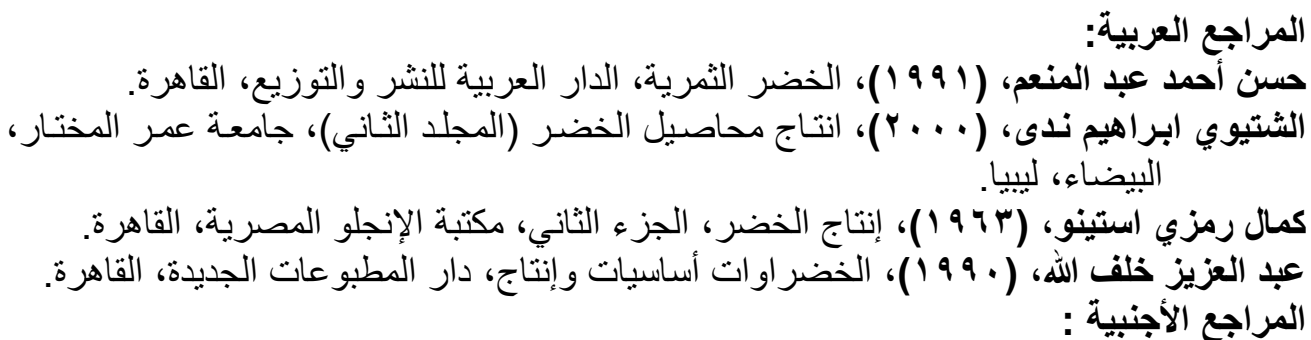

Arthey, V.D. (1975). Quality of horticultural products. Butterworths, London. 228 p.

Barton , L.V. and Fine, J. M. (1957). The effect of qibberellic acid on disease control - A bstr . in plant physiol . ( Hort. Abstr. (27) : 3059 ) .

Cook, A.R. (1957). Influence 1, 2, 4- D on the uptake of natrient from the soil, weeds $5: 25$, ( Hort . Abstr. (27): 2039).

Derevschukov, S.N., Zharavkova G.P. (2006). Legume crops: breeding and growing peculiarities. G: Potato and vegetables. N-5.C. 25. Mosqow.

Jeffrreys, D.B. (1964). The nature of growth in hibition in Lycopersicon esaulentum plants Treated compounds. Diss . Abstr . 24: 3071 (Hort. Abstr. (34): 3995).

Lorenz, O.A. and Maynard, D.N. (1980). Knott, S handbook for vegetable growers. Wiley Interscience, N.Y. 390 P.

Maier, I. and Others (1962). Effect of qibberellin treatment on some vegetable plants. Russian. French. English summaries Nur, Sti inst, Aqron. N. Balcescn, Ser 6: 287-300 (Hort. Abstr. (34): 6669).

Murphy, H.J. (1957). Growth regulater help snap bean production, Me . fn. Res , s ( 1 ) : 17 - 18 (Hort. Abstr. (27): 3983).

Miroshnikova, M.P. (2007). Seed breeding of vegetable bean. G: Potato and vegetables. N-4. C. 19. Mosqow.

Osipova, G.S., Kozhemjakov, A.P., Belobrova, S.N. and Thalean Haditha. (2007). Processing of seeds of vegetable haricot by biologicals. G; Potato and vegetables. N-6. C. 25. Mosqow.

Pakina, O.V. (2006). Estimation of plants diversity and breeding of bean,s varieties for Siberia. G: Potato and vegetables. N-5.C.26. Mosqow.

Sergeeva, I.I. (2007). Investigation of the nitrogen nutrition of plants during application of growth stimulators and bacteriological preparations. G;Potato and vegetables. N-5.C.38. Mosqow.

Wills, R.H.H., T.H. Lee, D. Graham, W.B. McGlasson and E.G. Hall. (1981). Postharvest. an introduction to the physiology and handling of fruit and vegetables. Granada, London. 163p.

\section{INFLUNCE OF APIREN HORMONE (IBA AND MATRIALS OF NITROGEN) ON GROWTH FBWERING AND DEVELOPMENT OF PHASEOLUS VULGAVIS, L.}

Fayoum J. Agric. Res. \& Dev., Vol.23, No.2, July, 2009 


\section{Ibrahim AL Shtewi}

Faculty of Agriculture, University of Alfurat

\section{ABSTRACT:}

The Current research paper Was Carried out using Four Varieties of phaseolus Vulgaris,Ll. ie .Giza, Iyolomska ,Strike And lo col variety .

The aim of This study Was to reveal:

The affect of apiren hormon on the growth, early fbwering end pollination of flowers of the ph. Vulgoris, 1 .

Two different concentrations of the hormon were employed: $5 \mathrm{ppm}$ ond $10 \mathrm{ppm}$. The Hormon was sprayed Twic. The first time at the emergen ce of the Faurth Tru leave. The second time at the fully opened flowers.

All the needed processes were carried on as recommended.

The Results of The Preent Research Showed That the Secand ancentration i.e. $10 \mathrm{ppm}$ for the used Hormon was the best. The Respon es of two varieties i.e Strike ond Igolomska. The respane the of tow verieties i.e strike and Igolomska were clearly manifested.

Fayoum J. Agric. Res. \& Dev., Vol.23, No.2, July, 2009 